\title{
Active Particle Condensation by Nonreciprocal and Time-delayed Interactions
}

\author{
Mihir Durve ${ }^{12}$, Arnab Saha ${ }^{3}$, and Ahmed Sayeed ${ }^{3}$ \\ 1 Department of Physics, Università degli studi di Trieste, Trieste, Italy 34127 \\ 2 The Abdus Salam International Centre for Theoretical Physics, Trieste, Italy 34151 \\ 3 Department of Physics, Savitribai Phule Pune University, Pune, India 411007 \\ Received: date / Revised version: date
}

\begin{abstract}
We consider flocking of self-propelling agents in two dimensions, each of which communicates with its neighbors within a limited vision-cone. Also, the communication occurs with some time-delay. The communication among the agents are modeled by Vicsek-rules. In this study we explore the combined effect of non-reciprocal interaction (induced by limited vision-cone) among the agents and the presence of delay in the interactions on the dynamical pattern formation within the flock. We find that under these two influences, without any position-based attractive interactions or confining boundaries, the agents can spontaneously condense into 'drops'. Though the agents are in motion within the drop, the drop as a whole is pinned in space. We find that this novel state of the flock has a well defined order and it is stabilized by the noise present in the system.
\end{abstract}

PACS. XX.XX.XX No PACS code given

\section{Introduction}

The co-operative motion of self-propelling individuals or agents, ranging from cellular and colloidal scales [1,2, 3, 4, 5, 6, 7, 8, 9, 10, 11 to the scales of the flocks of macroscopic living entities (e.g. birds, fishes, locusts etc.) [12,13, 14, 15, 16, 17, 18, 19, 20 depends on their internal degrees of freedom that consume and dissipate energy to their local environment in far from equilibrium conditions. The collective dynamics of such active systems often leads to features (e.g. dynamic pattern-formations and swarming) 21, $22,23,24,25,26,27$ which are not achievable in passive systems at equilibrium, or even in systems driven away from equilibrium by external fields. In recent times the research on collective dynamics of self-propelling individuals is contributing significantly to our current understanding of physics of living systems $28,29,30,31,32,33$.

In many collections of living agents on the move the main concern is to maintain the speed and avoid collisions with other nearby agents. Both of these requirements are achievable to an extent by adopting a simple rule, such as 'move as your neighbors do' - that is, each agents attempts continuously to align its heading (direction of motion) to the average heading of its neighbors. Vicsek et al. 21 proposed a model of velocity alignment which incorporates this behavior. This model manifests some of the important characteristics of natural flocks - such as

Correspondence to: arnab@physics.unipune.ac.in Correspondence to: sayeed@physics.unipune.ac.in ordered collective motion (e.g. band formation) for high agent densities and low noise strengths, and uncorrelated motion for low agent densities and high noises. Some recent reviews on this topic are 34,35 .

Two important features of inter-agent interaction are: (1) time delay and (2) reciprocity (or its absence). All the communications among agents in nature must have some finite amount of delay. It is determined both by the speed of the signal used to communicate and the time taken by an agent to process the information regarding the communication. The communication and interaction among the agents are often non-reciprocal in the sense that the influence of, say agent $i$ on agent $j$ is in general different from that of the agent $j$ on agent $i$. A simple example is human pedestrian motion [36]. Each individual in a pedestrian crowd usually notices a person in front of him, but is not noticed in turn by that person.

The model we have studied here incorporates both communication delay and non-reciprocity. Time delay is introduced in the dynamics of the agents by making agents 'slow-to-notice' the velocities of their neighbors by one time step (i.e., an agent uses neighbors' velocities which are 'out-of-date' by one time-step to determine its next step). Non-reciprocity is introduced by assigning a limited (i.e. $\leq 2 \pi$ ) angular range of interaction to every agent. This limited angular range is termed as 'vision-cone'. As an example of how a vision cone can induce non-reciprocal interaction, one may consider a typical situation where, at time $t$, the agent $j$ affects the motion of agent $i$ because $j$ is within the vision cone of $i$. But at the same time, it 
may happen that $i$ is not in the vision cone of $j$, and thus $i$ does not affect the dynamics of $j$. This is illustrated in Fig. 1 .

In this work these two common and important features of many natural flocks are considered together to investigate their interplay. To our knowledge, it is by and large unexplored previously, although each of the features has been explored separately in recent studies. For example, the role of time-delay has been explored by 37 where phototactic robots are found to form metastable clusters depending on their inherent sensory delay (delay to sense the optical signal) and the intensity of the signal. Here the delay is associated with the robots to sense an external signal. Time delay can also be associated with sensing signals originated within the flock, i.e. the internal signal. For example, an individual agent of a flock can be slow enough to respond to a signal coming from its neighbour. This causes communication-delay [38, 39, 40, 41. It has been shown that in presence of a noise induced transition from translatory to rotatory motion of agents [42], the time-delayed communication among them can introduce further instabilities where the harmonically interacting agents can form dynamic clusters or swarms, with a high degree of polarity 38 .

Reciprocity is the norm in the interactions among passive particles (atoms, molecules, colloidal particles etc.), though there are evidences of non-reciprocal effective physical interactions in out-of-equilibrium conditions. For example, when a system of particles is embedded in and interacts with each other via a moving, out-of-equilibrium medium, the effective interaction among the particles can be non-reciprocal. In particular, diffusiophoretic and optical forces within colloids [43, 44, 45, 46, effective interaction among colloidal particles within a flowing solvent 47 48, 49, shadow or wake mediated forces between particles within flowing plasma [50,51,52 are examples for nonreciprocal, physical interactions. But in such cases one can recover the reciprocity by considering the medium and the particles together.

In contrast to many passive systems, interactions among the agents of a flock are often non-reciprocal [53,54, a fact that is barely appreciated in the much of the literature on active systems. The issue of non-reciprocity has been incidentally encountered (but not addressed specifically) in a few studies that explore the role of limited vision-cone of active, interacting agents in a flock. Angular restriction on the reorientation of an individual agent within a flock alone can facilitate ordering $[55$ and can also affect the thermodynamic character of the order-disorder transition occuring within the flocks [56. It was also observed that angular restriction on interaction neighborhood in Vicsek model can reduce time required to reach ordered state from a disordered state [57. Recently it has also been shown that vision-cone can induce complex, selfassembled structures within the flock of self-propelling, memory-less agents, communicating among themselves by position-based, attractive interactions [58].

In contrast to the earlier studies, here we introduce the delay in communication among the agents and the angu- lar restriction together, by adapting the Vicsek model. We report here emergence of an instability leading to what we call the drop state, which is similar in some respects to the patterns discussed as 'absorbing states of the flock with frozen fluctuations' in reports [59,60,61]. In particular our study shows that the agents with narrow vision cones and delayed responses spontaneously condense and confine themselves within small regions, eventually forming a few randomly positioned, dense clusters, which we call 'drops'. Within such a drop, agents are in motion but the drop as a whole is almost immobile, with only small fluctuations in the position of its center of mass. Also, the average angular momentum of the agents within the drop fluctuates about zero, which means these drops do not have votex-like dynamics either. The small size and immobility of the drop is sustained because individual agents perform a sequence of correlated, large-angle turn-arounds, effectively confining themselves to the drop. Importantly, we find that the drops are stable only if there is a finite amount of noise present in the interaction among the agents. The drop disperses if the noise strength falls below a critical value. In the following sections we describe our model and report the results in detail.

\section{Model}

We add communication-delay among the agents together with non-reciprocity to a collection of Vicsek-agents. The non-reciprocity is induced by the vision-cone (see the Appendix), i.e. the interaction neighborhood of an agent which is not a circle centered around that agent (as it was in original Vicsek model), but a sector of this circle, as illustrated in Fig. 1. The neighborhood sector $\mathrm{S}_{i}$, which we call the vision-cone, has an opening angle $2 \phi$ and is centered about the direction of velocity of the $i^{\text {th }}$ particle. We shall call the half opening angle $\phi$ as the 'view-angle', which can vary from 0 to $\pi$. For $\phi=\pi$, this model reduces to the standard Vicsek model [21,22].

At time $t, N$ number of agents have positions and velocities $\left\{\mathbf{r}_{i}(t), \mathbf{v}_{i}(t)\right\}_{i=1}^{N}$. We calculate the average velocity (denoted in square brackets below) of the agents within the vision-cone $S_{i}$ of the $i^{\text {th }}$ agent as

$$
\left[\mathbf{v}_{\mathrm{vc}}(t)\right]_{i}=\frac{1}{N_{i}} \sum_{j \in \mathrm{S}_{i}} \mathbf{v}_{j}(t),
$$

where $N_{i}$ is the number of agents within the vision-cone $S_{i}$ and the subscript 'vc' denotes vision-cone. Now we find the velocity of $i^{\text {th }}$ agent at time $t+1$ as,

$$
\mathbf{v}_{i}(t+1)=v_{0} \mathcal{R}_{\eta}(\theta) \circ\left[\hat{\mathbf{v}}_{\mathrm{vc}}(t)\right]_{i},
$$

where the over-hat ${ }^{\wedge}$ indicates unit vector. $\mathcal{R}_{\eta}(\theta)$ is the rotation operating (denoted by ' $\circ$ ') on the unit vector $\left[\hat{\mathbf{v}}_{\mathrm{vc}}(t)\right]_{i}$ to rotate it by an angle $\theta$. The angle $\theta$ is a random variable uniformly distributed over the interval $[-\eta \pi, \eta \pi]$, where $\eta$ is the strength (i.e., amplitude) of the noise that can be varied from 0 to $1 . v_{0}$ is the constant speed of the 
agents. It should be noted here that the neighborhood $S_{i}$ is as seen at time instant $t$.

Though we calculate $\mathbf{v}_{i}(t+1)$, we update the positions with $\mathbf{v}_{i}(t)$, i.e.,

$$
\mathbf{r}_{i}(t+1)=\mathbf{r}_{i}(t)+\mathbf{v}_{\mathbf{i}}(t) \Delta t
$$

where $\Delta t=1$. Thus positions are updated with velocities which lag by one time step. This introduces the delay in 'response' of an agent to the motion of its neighbors.

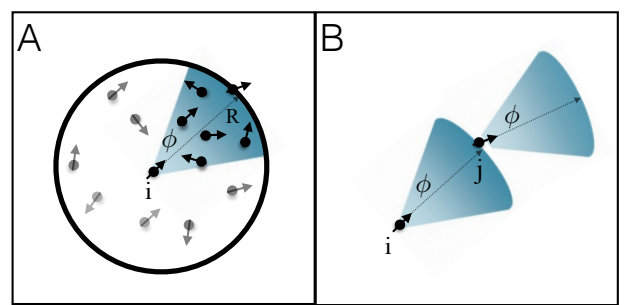

Fig. 1. (A) The neighborhood $\mathrm{S}_{i}$ (blue shaded) of the $i^{\text {th }}$ agent. The $i^{\text {th }}$ agent is shown at the center of a circle of radius $R$ and the neighborhood $\mathrm{S}_{i}$ is the blue sector of the circle. The black dots with arrows as heading directions indicate the agents lying within the neighborhood (including the particle at the center of the circle), and the gray dots with arrows as heading directions indicate agents outside it. The view-angle $\phi$ is the half opening angle of the neighborhood at the center. (B) An example of non-reciprocal configuration of agents $i$ and $j$, where $i$ interacts with $j$ but not the other way round.

The degree of order in the collective motion of the particles is measured by a scalar order parameter $\bar{\psi}$ defined as,

$$
\bar{\psi}=\left\langle\frac{1}{N v_{0}}\left|\sum_{i=1}^{N} \mathbf{v}_{i}(t)\right|\right\rangle,
$$

where the angular bracket denotes the average over multiple realizations and the steady state time average over a time-window $\tau . \bar{\psi}$, in the perfectly ordered state (when all the particles are moving in the same direction) becomes unity and in the completely disordered state (when the directions of motion are completely random) becomes zero, in the limit of $N \rightarrow \infty$. In this report we use the phrase 'ordered state' to mean the stationary state of the system for which $\bar{\psi}>0$ in the limit of $N \rightarrow \infty$.

\section{Simulation Details}

The simulations were carried out in two-dimensional square box of size $L$. At time $t=0, N$ agents are placed randomly and uniformly in the square box. The positions of the agents are denoted by $\mathbf{r}_{i} ; i=1,2 \ldots N$. The bulk agent density is given by $\rho=N / L^{2}$. For this study we carried out simulations with $N=144,256,400$ and 576. Initial directions to the agents $\left(\theta_{i} ; i=1,2 \ldots N\right)$ are assigned randomly and uniformly in the range $[-\pi$ to $+\pi] . \theta_{i}$ are measured in a fixed frame of reference. All the agents have the same, constant speed denoted by $v_{0}$. Then at each simulation time step the positions $\mathbf{r}_{i}(t)$ and velocities $\mathbf{v}_{i}(t)$ of all the agents are updated simultaneously according to the model described in the section 2. The periodic boundary conditions are applied in both directions.

Throughout the study the following parameters are fixed: the radius of interaction $R=1.0$, bulk number density of agents $\rho=1.0$, and the speed of the agents $v_{0}=0.5$. The steady state quantities are obtained by averaging over 20 independent realizations as well as over time $\tau$. Each realization is of length $10^{5}$ time-steps, out of which we discard the initial $2 \times 10^{4}$ time-steps and use the rest $8 \times 10^{4}$ time-steps for steady-state time-averaging. Here onwards all angles are given in units of $\pi$ radians. The other specific details are provided in respective figure captions.

\section{Results and Discussions}

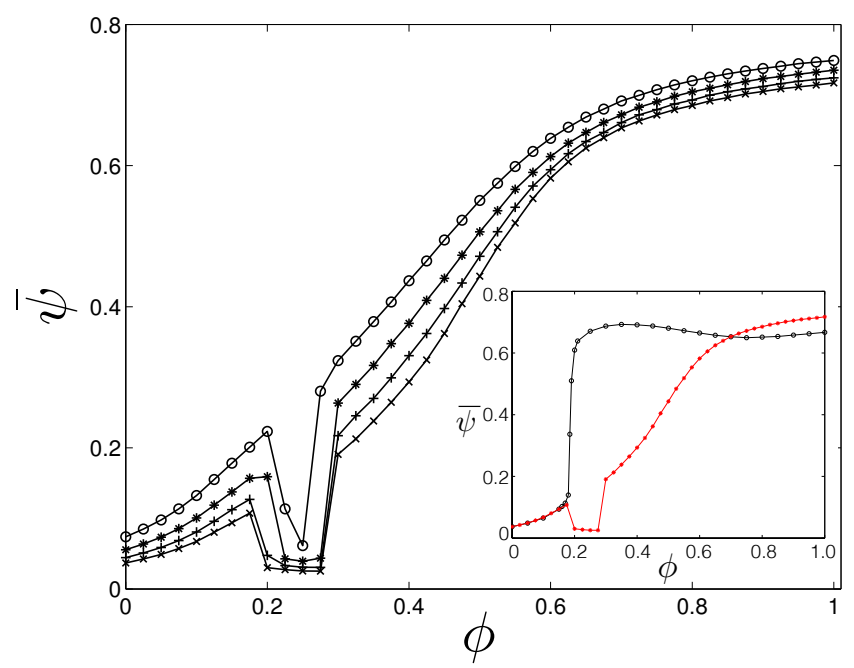

Fig. 2. Plot of order parameter $\bar{\psi}$ vs view-angle $\phi$. The circle, star, plus and cross corresponds to system sizes $N=$ $144,256,400,576$ respectively, and the noise strength $\eta=0.3$. Inset : Plot of order parameter $\bar{\psi}$ vs view-angle $\phi$ with (red star) and without (black, open circle) delay, $\mathrm{N}=576$.

The main figure of Fig. 22 shows steady-state average (over time and multiple configurations) of Vicsek order parameter $\bar{\psi}$ as a function of the view-angle $\phi$ for four different system sizes. Here we see the most remarkable anomalous behavior of the system - the order parameter $\bar{\psi}$ dips to a value close to zero around $\phi=0.28$, and then again recovers to higher value at lower $\phi$ values. As the system size increases, the range of $\phi$ over which the dip exists widens. Within this anomalous range of $\phi(\approx 0.20$ to 0.28 ), for $N=576$, the value of the $\bar{\psi}$ is slightly lower than what is expected for a completely disordered state (which yields a small non zero value of the order of $10^{-2}$ due to finite system size). As we shall describe below, in this range of $\phi$, the system is indeed not in a disordered state, but in a remarkable new, ordered state where the 
agents spontaneously confine themselves in a small, almost immobile cluster which we will refer as a drop. Vicsek order parameter $\bar{\psi}$ for this drop is as close to zero as it is for completely disordered state of the agents. Therefore $\bar{\psi}$ is unable to capture the difference between this drop state and a completely disordered state. Later we define a new order parameter to quantify this order.

In the inset of Fig. 2 we have shown a comparison of the system behaviour in the presence and absence of delay. In this comparison all the parameter values in the two cases are the same, and the system without delay is simulated using the model rules described in [56]. Here we see that in the absence of delay the drop state, which is marked by a $\bar{\psi} \approx 0$ region around $\phi=0.24$, is absent. This directly manifests the essential role played by the delay in producing the drop state. Also in both cases, taking the standard Vicsek dynamics limit with $\phi=1$, we obtain the value of order parameter $\bar{\psi} \approx 0.7$. It indicates the system is in ordered state. Therefore at this limit, in both cases (i.e., with and without delay) we observe an ordered state with agents moving in band-like structures (not shown here, but discussed in [56]).
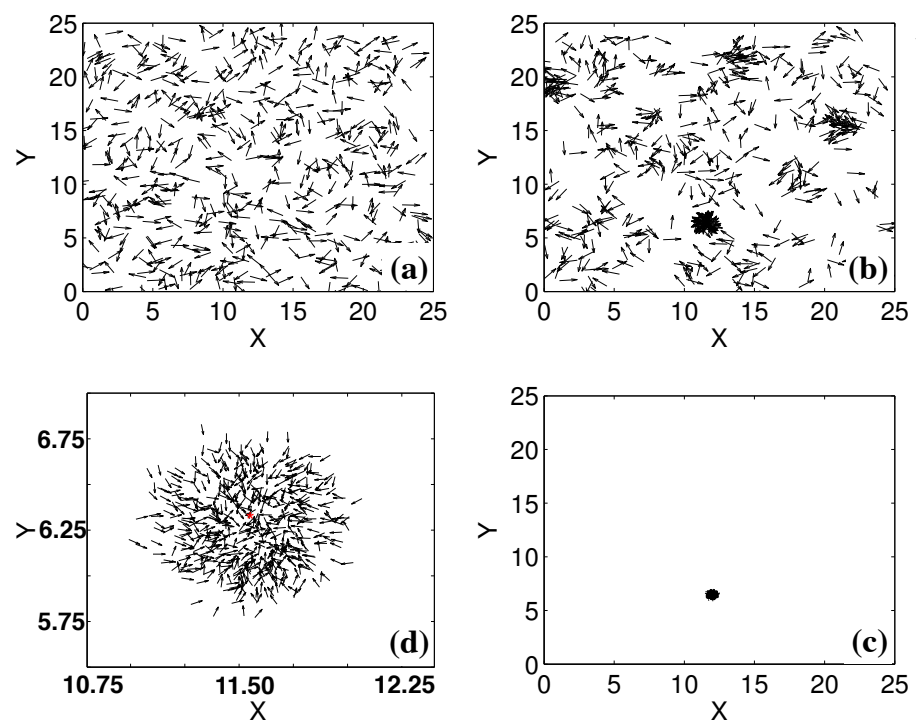

Fig. 3. Snapshot of the system at various time instances (a) 0 , (b) 168 , (c) 4500 . Arrows indicates the directions of the motion of the agents. (d) shows a zoomed view of the stable drop at $t=4500$. The centre of mass of the drop is indicated by a red asterisk. The parameters are $N=576, \eta=0.3$.

We now describe the emergence of this new kind of condensed state with a sequence of snap-shots of agent distribution within the box, shown in Fig. 3. Here we set $\phi=0.24$, because this is the mid-point of the $\phi$-range over which the drop state is stable for the system size $N=576$. The initial distribution in Fig 3 a shows that the agents are homogeneously distributed and their directions are uniformly random. As the time progresses transient nucleation of agents occurs at several places within the system, shown in Fig $3 \mathrm{~b}$. With time we observe emergence of one stable nucleus shown in Fig $3 \mathrm{c}$, which acts as a sink for the agents passing close-by. Once a drifting agent gets within one step-length of the sink it is irreversibly captured, and this process continues until all the agents condense into a single drop of size roughly equal to one step-length. This whole process can clearly be seen in the movie provided in Supplementary Material. For the system size used for this report we obtain only a single drop. But we have found that for larger system sizes multiple such drops are formed spontaneously, which are stable, well-separated and randomly placed within the system 62 . Fig. 3 d shows the zoomed view of the stable drop state of the system. The arrows indicate the instantaneous velocity directions of the agents. The directions appear to be random, but there is a pronounced radially inwards bias. This implies that there is no significant net tangential velocity component. This prevents vortex-like collective motion within the drop.

The absence of vortex-like motion is also evident from Fig 4. This figure shows the variation of average angular velocity $\omega_{z}(t)=\langle\mathbf{r}(t) \times \mathbf{v}(t)\rangle$ with time. Here the plot is for a single realization and the averaging is over all the agents at time $t$. Here we see that $\omega_{z}$ fluctuates rapidly about zero in time. This indicates that the agents are equally likely to have positive or negative angular velocity. Therefore the average angular velocity of the collection of the agents within the drop becomes approximately zero.

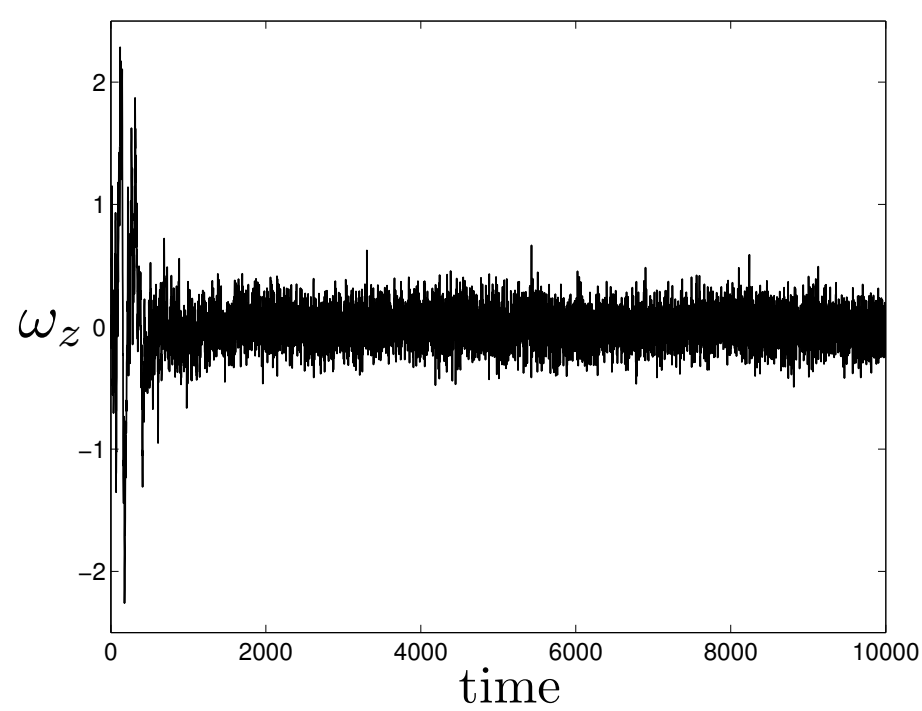

Fig. 4. Average angular velocity $\omega_{z}$ vs $t$ plot for a single realisation. Parameters are $N=576, \eta=0.3, \phi=0.24$.

The local number density $\rho_{\text {loc }}(r)$ (where $r$ is the radial distance of the agents from the center of the drop) of the agents within the drop is not uniform. This is the number of agents per unit area at a distance between $r$ and $r+d r$. It has a maximum at a certain radial distance from the center. This is clear from Fig.5, where we see that the steady state density of the agents along the radius of the drop peaks at a distance of about 0.25 , which is about half the radius of the drop. The shape of the drop fluctuates in time, but the time-averaged shape is circular in steady state, as is shown in the Fig 5 inset. 


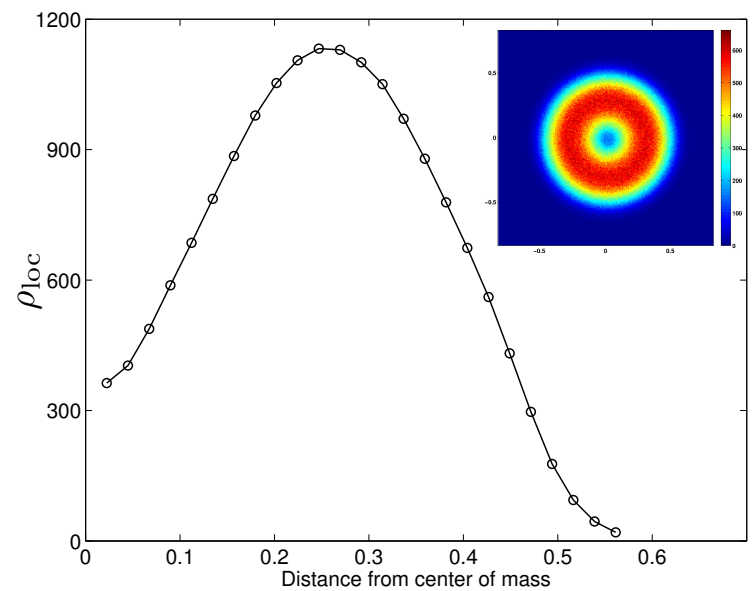

Fig. 5. Radial variation of local number density of the agents from the center of mass of the system with $N=576$ is plotted. In the inset, the $2 \mathrm{~d}$ distribution of the agents within the drop of the same system is shown in a $3 \mathrm{~d}$ plot where the $3^{\text {rd }}$ axis (i.e. color-axis) represents their density. The positions of the agents are measured from the center of mass of the system, placed at the origin in the plot. The density plotted here is for a single realization but averaged over time in steady state. The parameters are $\eta=0.3, \phi=0.24$.

We further studied the effect of the noise on the drop. The most remarkable feature of the drop is that it is stabilized by noise. The drop state is not stable when the noise strength is below a certain threshold. This is shown in Fig. 6 and Fig. 7. Fig 6 (a) shows the order parameter $\bar{\psi}$ against view-angle $\phi$ for different noise strengths. The dip in the order parameter (which results from the drop formation as described before) is most pronounced for a noise $\eta=0.3$, and is absent for $\eta \leq 0.20$ and $\eta>0.35$. Fig.6(b) presents the phase diagram over the intervals for view-angle $\phi \in(0,0.6)$ and noise strength $\eta \in(0,0.4)$, which manifests condensation of agents over a considerable range of the parameters $\phi$ and $\eta$.

This condensation is also apparent in Fig. 7, where we have shown the variation of radius of gyration $R_{g}$ as a function of noise strength $\eta$. Here $R_{g}$ is defined as follows:

$$
R_{g}=\left\langle\left[\frac{1}{N} \sum_{i=1}^{N}\left(\mathbf{r}_{i}-\mathbf{r}_{\mathrm{cm}}\right)^{2}\right]^{1 / 2}\right\rangle \text {. }
$$

In the above definition $\mathbf{r}_{\mathrm{cm}}$ is the centre of mass of the system and the angular brackets indicate averaging over the steady state time-window $\tau$ and over multiple realizations. We expect $R_{g}$ to collapse to a small value (comparable to the size of the drop) in the drop state, and thus providing another signature of the transition to the drop state. In this plot shown in Fig 7 the drop-state of the system is reflected as a small radius of gyration $\left(R_{g} \approx 1.25\right)$, which persists over a noise range of $\eta=0.20-0.35$, and thus consistent with the observations in Fig.6. In a different context, Chepizhko et. al. 63 observed that a finite amount of noise is required to stabilize order in the collective motion of agents in heterogeneous medium.
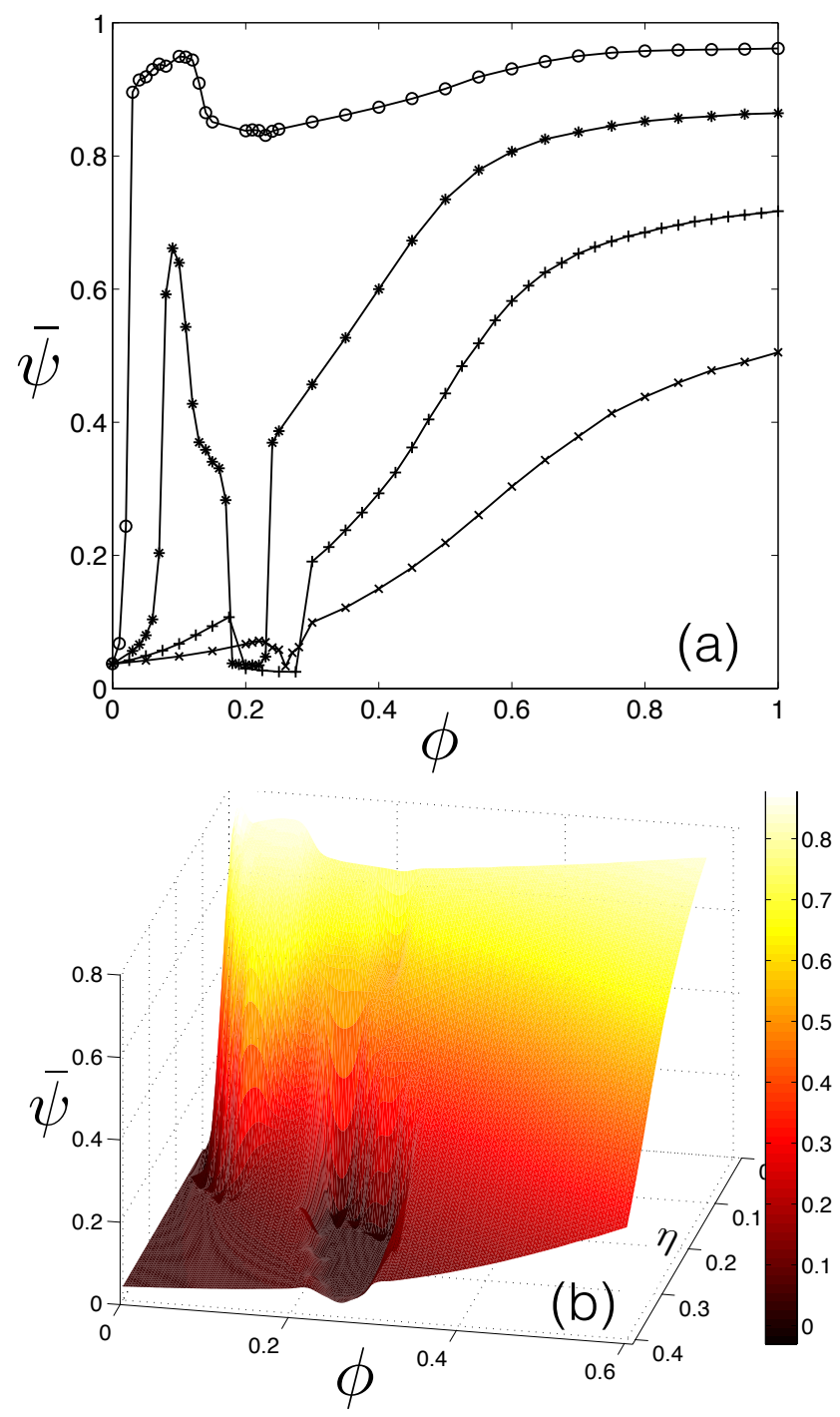

Fig. 6. (a) Behavior of the order parameter $\bar{\psi}$ with viewangle $\phi$ for various noise strengths. The circle, star, plus sign and cross corresponds to noise $\eta=0.1,0.2,0.3,0.4$ respectively. Here $N=576$. (b)Phase diagram: variation of the order parameter $\bar{\psi}$ (colour axis) with view-angle $\phi$ and noise-strength $\eta$. The valley, shown as the dark-shaded region, indicates the parameter space for the condensation.

Now we discuss how velocity correlations of the agents vary with the view angle $\phi$ for a given noise strength $\eta$, in the range where the system is in the drop state. In steady state, we calculate average velocity autocorrelation $\bar{\chi}$, which is defined as,

$$
\bar{\chi}=\left\langle\frac{1}{N} \sum_{i} \frac{\mathbf{v}_{i}(t+1) \cdot \mathbf{v}_{i}(t)}{\left|\mathbf{v}_{i}(t+1)\right|\left|\mathbf{v}_{i}(t)\right|}\right\rangle,
$$

where the angular bracket implies steady state time average as well as the average over multiple realizations. $\bar{\chi}$ estimates the cosine of the angle $\Lambda$ between the directions $\hat{\mathbf{v}}_{i}(t+1)$ and $\hat{\mathbf{v}}_{i}(t)$ of two consecutive moves of the same agent. We call $\Lambda$ the average turn-around angle. $\bar{\chi}$ is plotted with varying view-angle in Fig, 8 . We see that 


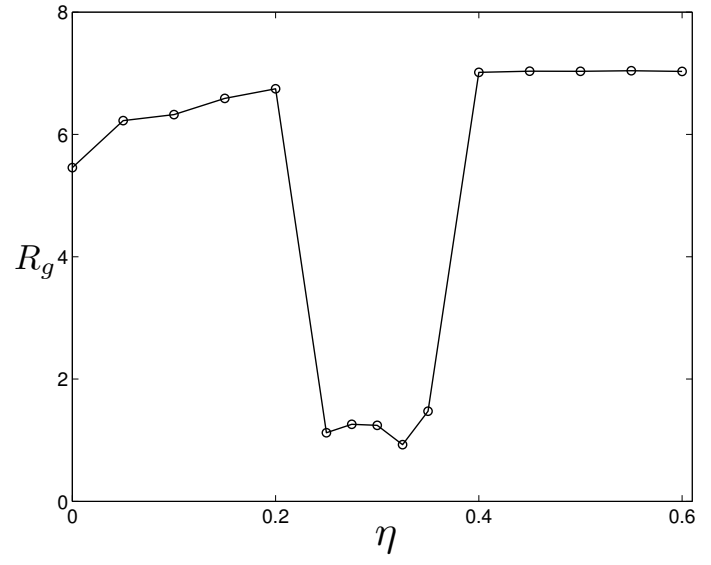

Fig. 7. Radius of gyration $R g$ vs noise $\eta$ plot. The parameters are $: N=576, \phi=0.24$.

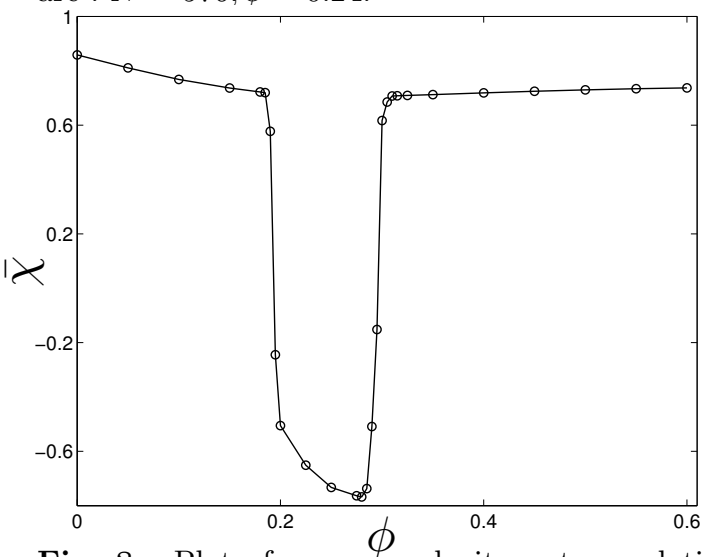

Fig. 8. Plot of average velocity autocorrelation $\bar{\chi}$ vs viewangle $\phi$. Here $N=576, \eta=0.3$.

$\bar{\chi} \approx-0.75$ in the drop state (view-angle range: 0.18 to 0.3 ) and otherwise $\chi \approx+0.8$. Thus in the drop state $\Lambda \approx 2.43$ and otherwise $\Lambda \approx 0.65$. Also, we define a velocity crosscorrelation function, between the instantaneous velocity of the $i^{\text {th }}$ agent and the average velocity of the agents within its vision-cone, $\left[\mathbf{v}_{\mathrm{vc}}\right]_{i}$ (defined earlier) as,

$$
\bar{\alpha}=\left\langle\frac{1}{N} \sum_{i} \frac{\mathbf{v}_{i}(t) \cdot\left[\mathbf{v}_{\mathrm{vc}}(t)\right]_{i}}{\left|\mathbf{v}_{i}(t)\right|\left|\left[\mathbf{v}_{\mathrm{vc}}(t)\right]_{i}\right|}\right\rangle .
$$

The angular bracket indicates the average over time in the steady state and over multiple realizations. $\bar{\alpha}$ is the average cosine of the angle $\Gamma$ between the instantaneous velocity $\mathbf{v}_{i}(t)$ of the $i^{\text {th }}$ agent and $\left[\mathbf{v}_{\mathrm{vc}}(t)\right]_{i}$. Thus $\bar{\alpha}$ is an order parameter that measures the extent of correlation in motion between an agent and its neighbours. As we stated earlier, Vicsek order parameter does not capture this correlation in the motion of agents in the drop state. But as we show here $\bar{\alpha}$ clearly quantifies this correlation, as presented in Fig 9 which gives variation of $\bar{\alpha}$ with the view-angle $\phi$. Here we see $\bar{\alpha}$ has a sharp dip within the range $0.18<\phi<0.30$, carrying the signature of the drop formation. The value $\bar{\alpha} \approx-0.85$, which corresponds to $\Gamma \approx 2.58$. This clearly shows an agent is moving mostly in a direction opposite to the average direction of its neighbours in the drop state (the exact opposition in motion, i.e., $\Gamma=\pi$ will give $\bar{\alpha}=-1$ ). This 'go against the flow' is interesting when contrasted with usual Vicsek ordered state where agents tend to 'go along the flow'. The behaviour of $\bar{\alpha}$ beyond the drop phase (i.e., outside the range $0.18<\phi<0.30)$ can be explained as follows. For very small view-angle $\phi$ the vision-cone of an agent contains no other agents, so effectively the system is a collection of non-interacting agents. And thus $\bar{\alpha}$ reduces to velocity self-correlation of an agent at a given instant of time, and therefore takes the value unity. For larger $\phi$ values the system attains significant Vicsek ordering, in which case the angle between an agent's velocity and the average velocity in the vision cone is small, i.e., $\Gamma \approx 0.63$ corresponding to $\bar{\alpha} \approx+0.81$.

Taking together the observations in Fig, 8 and Fig. 9 we can explain the process that spontaneously confines the agents within the drop as follows. Each agent finds other agents in front of it within a narrow cone and obtains their average heading (i.e., $\hat{\mathbf{v}}_{\mathrm{vc}}$ ), which happens to be largely opposed (i.e., by an angle $\approx 2.58\left(=148^{\circ}\right)$ ) to its current direction. But the agent persists with its current heading for one time step due to the delay, and then it turns around by a large angle (average turn-around angle $\Lambda \approx 2.43\left(=139^{\circ}\right)$ ), remembering the average orientation of its neighbours in the previous step. Such successive large turn-arounds effectively confine the agents to a small region (i.e, the drop), with a linear size approximately equal to one step-length.

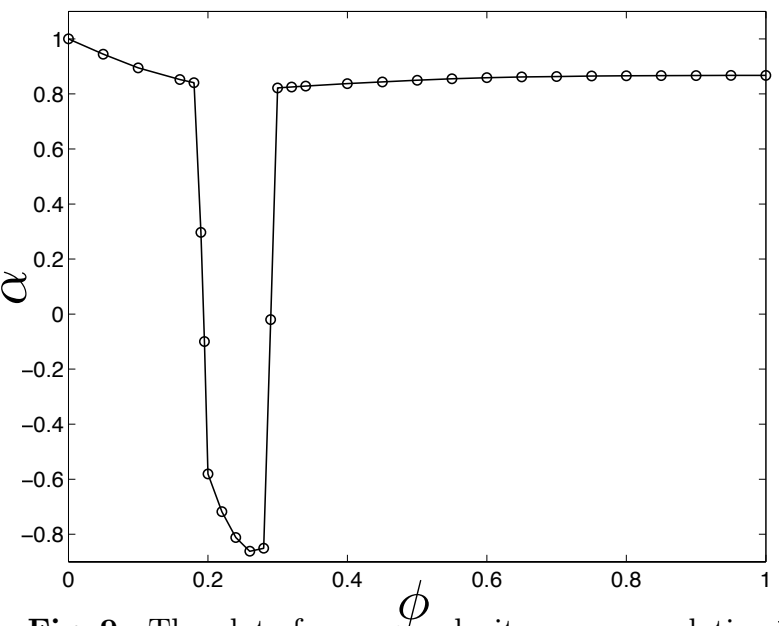

Fig. 9. The plot of average velocity cross-correlation $\bar{\alpha}$ versus view-angle $\phi$. Here $N=576$ and $\eta=0.3 \pi$.

\section{Finite Size Effects}

Here we discuss the finite size effects on the condensation, i.e. the effect of varying the number of agents $N$ keeping the density same. We have seen that the formation of the drop state is indicated by the collapse of $R_{g}$ to a small value. Therefore we have measured the variation of $R_{g}$ against the noise strength $\eta$ and the view angle $\phi$ for three system sizes $N=1000,1500,2000$. This data is presented 
in Fig 10 . Here we clearly see that the drop state persists as we increase the system size, and the finite size effects on the ranges of $\eta$ and $\phi$ are very small. But as we increase the system size we find that multiple drops can form. In the simulations for the data presented in Fig. 10 we found two drops in each case. The $R_{g}$ values are calculated as weighted averages over the two drops in each case.
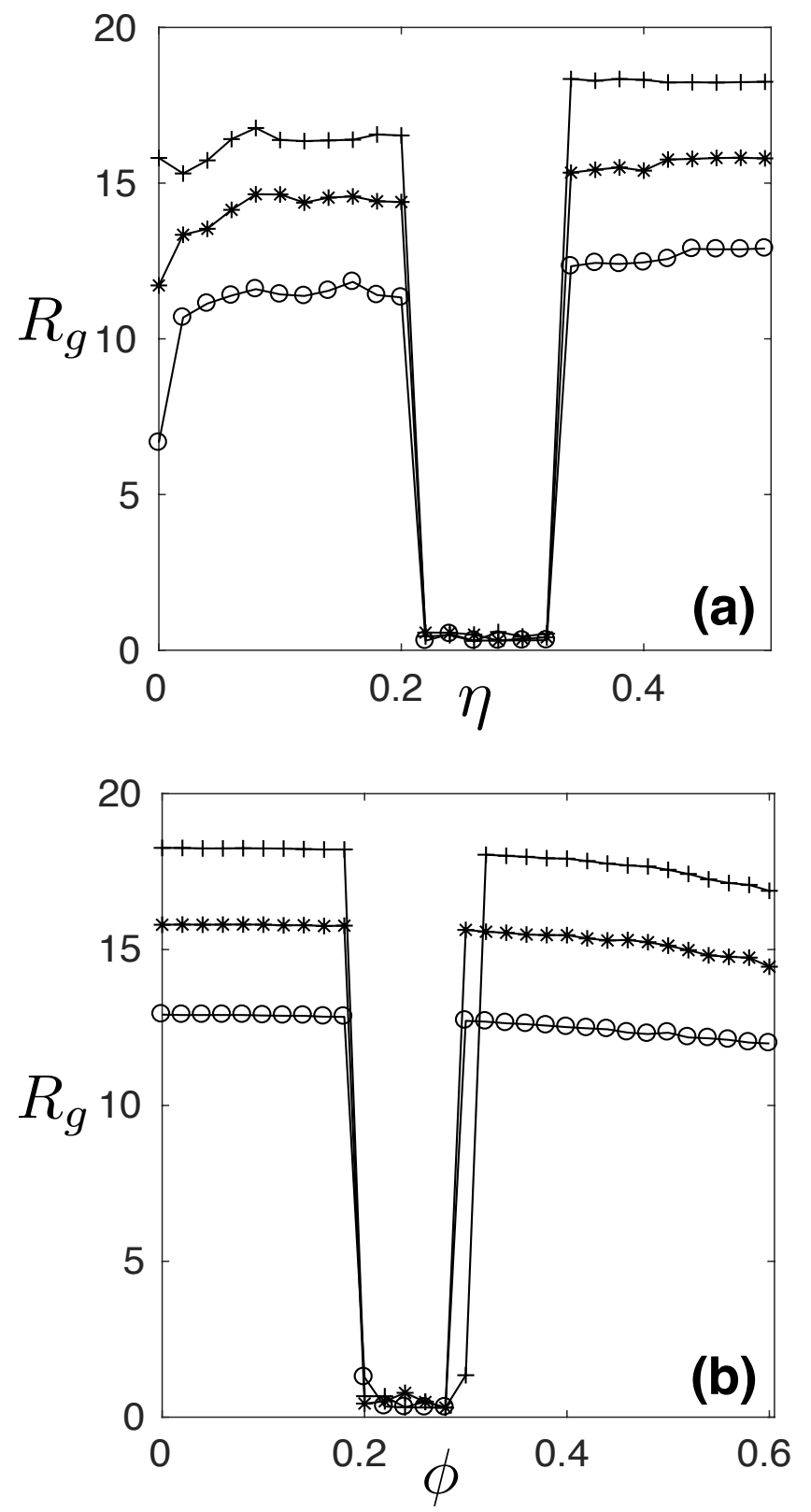

Fig. 10. (a) Radius of gyration $R_{g}$ vs Noise $\eta$. The symbol circle,star,plus corresponds to system size $N=1000,1500,2000$ respectively. View-angle $\phi=0.24$. (b) Radius of gyration $R_{g}$ vs View-angle $\phi$. The symbol circle,star,plus corresponds to system size $N=1000,1500,2000$ respectively. Noise $\eta=0.3$.

\section{Summary and Conclusions}

We have explored the collective behavior of self-propelled particles or agents where every agent communicates with its neighbors through a vision cone defined by angular as well as radial limits and with a time-delayed response to their motion. Angular limit on the vision-cone introduces non-reciprocity in the interaction among the agents. Even in the absence of any attractive interaction among the agents, the combination of the non-reciprocity and the delay are enough to produce a remarkable state where agents spontaneously condense into a dense drop. The drop remains essentially pinned in space. It occurs within a particular parameter space of the opening angle of the visioncone and the noise strength of the agents, for a given bulk agent-density of the system. In that parameter space the motion of the agents consists of high-angle turn-arounds, which effectively confine the agents to the drop. Within the drop the number density of the agents is radially nonuniform and no vortex like motion is observed. Importantly, the drop is stabilized within a finite band of noise. With noise below than a certain strength the drop becomes unstable. The drop is destabilized also at higher noise strength.

Now we conclude with a brief comparison between the phenomenon observed in this study and the aggregation of active particles in the context of motility induced phase separation (MIPS), which has been a subject of recent research interest 64. In both cases we observe aggregation of active particles or agents, but there are some crucial differences between the mechanism of the formation of the clusters. In MIPS, a positive feed back between the slowing-down-induced accumulation and accumulation-induced slowing down of active particles destabilizes the uniform density state, and results in spontaneous formation of clusters coexistent with a gas-like phase. Repulsion or excluded-volume-interaction among the agents plays a crucial part in slowing down mechanism within a cluster generated by MIPS. But in our case, the agents are point-like objects, subject to Vicsek-like alignment. Moreover, in the aggregation we have observed (i.e, drop state), the non-reciprocal interaction combined with timedelayed comunication among the agents are essential. To our knowledge neither of which are present in the current theory of MIPS. Though it will be interesting to explore if one can generalize the theory of MIPS to accommodate the non-reciprocity and delay.

\section{ACKNOWLEDGMENTS}

We acknowledge financial support from Board of College and University Development, Savitribai Phule Pune University. This work was carried with HPC facilities provided by Centre for Development of Advanced Computing (CDAC) and also HPC facilities under the DST-FIST program at the Department of Physics, Savitribai Phule Pune University and HPC facilities provided by the I.C.T.P, Italy. One of the authors (Arnab Saha) acknowledges UGCFRP for the financial support. One of the authors (Mi- 
hir Durve) is thankful for graduate fellowship from the I.C.T.P. Italy and University of Trieste, Italy. Authors thank Debashish Chaudhuri and Sriram Ramaswamy for useful comments.

\section{Appendix : Vision-cone And Non-reciprocity}

Here we will discuss how a limited vision-cone can induce non-reciprocity in the inter-agent interactions. The interaction between $i^{\text {th }}$ and $j^{\text {th }}$ agents, namely $V_{i j}$ is nonreciprocal when $V_{i j} \neq V_{j i}$. For simplicity here we consider only two self-propelling agents, in zero noise limit, with directions of motion $\hat{\mathbf{v}}_{\mathbf{1}}, \hat{\mathbf{v}}_{\mathbf{2}}$ at time $t$. But the discussion here can be generalised to an arbitrary number of agents with finite noise.

When the distance between the agents are less than $R$, the directions of motion of the agents can be updated at $(t+1)$ as,

$$
\hat{\mathbf{v}}_{1}(t+1)=\frac{\hat{\mathbf{v}}_{1}(t)+\Theta\left(\phi-\delta_{12}\right) \hat{\mathbf{v}}_{2}(t)}{1+\Theta\left(\phi-\delta_{12}\right)}
$$

and

$$
\hat{\mathbf{v}}_{2}(t+1)=\frac{\hat{\mathbf{v}}_{2}(t)+\Theta\left(\phi-\delta_{21}\right) \hat{\mathbf{v}}_{1}(t)}{1+\Theta\left(\phi-\delta_{21}\right)}
$$

Here $\delta_{12}=\measuredangle\left(\hat{\mathbf{v}}_{1}, \hat{\mathbf{r}}_{12}\right)$ and $\delta_{21}=\measuredangle\left(\hat{\mathbf{v}}_{2}, \hat{\mathbf{r}}_{21}\right)$. Here $0 \leq$ $\left(\delta_{12}, \delta_{21}\right) \leq \pi$. $\Theta$ is the Heaviside step function i.e. $\Theta(x)=$ 1 when $x \geq 0$ and $\Theta(x)=0$ if $x<0$. If $\phi=\pi$, we recover Vicsek-like velocity alignment in zero noise limit. Using the representation

$$
\Theta(x)=\frac{1}{2}+\frac{1}{2} \lim _{q \rightarrow \infty} \tanh (q x)
$$

equations 89 can be rewritten as

$$
\hat{\mathbf{v}}_{1}(t+1)=\frac{\hat{\mathbf{v}}_{1}(t)+\frac{1}{2}\left(1+\lim _{q \rightarrow \infty} \tanh \left(q \sigma_{12}\right)\right) \hat{\mathbf{v}}_{2}(t)}{1+\frac{1}{2}\left(1+\lim _{q \rightarrow \infty} \tanh \left(q \sigma_{12}\right)\right)}
$$

and

$$
\hat{\mathbf{v}}_{2}(t+1)=\frac{\hat{\mathbf{v}}_{2}(t)+\frac{1}{2}\left(1+\lim _{q \rightarrow \infty} \tanh \left(q \sigma_{21}\right)\right) \hat{\mathbf{v}}_{1}(t)}{1+\frac{1}{2}\left(1+\lim _{q \rightarrow \infty} \tanh \left(q \sigma_{21}\right)\right)}
$$

where $\sigma_{12}=\phi-\delta_{12}$ and $\sigma_{21}=\phi-\delta_{21}$. Here $q$ is a positive parameter signifying the sharpness of the vision-cone boundary. Note that if agent " 2 " is within the vision-cone of the agent " 1 ", $\sigma_{12}$ is positive, and otherwise negative. Similarly, for the agent " 1 ".

For our purpose, we need the large $q$ limit (as our vision-cone boundaries are infinitely sharp) where, $\tanh \left( \pm q \sigma_{12}\right) \simeq \pm\left(1-2 \exp \left(-2 q\left|\sigma_{12}\right|\right)\right)$. Using this asymptotic expansion when agent " 2 " is inside the vision cone of "1", Eq. [11] becomes,

$$
\hat{\mathbf{v}}_{1}(t+1) \simeq \frac{1}{2}\left(\hat{\mathbf{v}}_{1}(t)+\hat{\mathbf{v}}_{2}(t)\right)+\frac{1}{4}\left(\hat{\mathbf{v}}_{1}(t)-\hat{\mathbf{v}}_{2}(t)\right) e^{-q\left|\sigma_{12}\right|}
$$

In Eq. 13 The first term is Vicsek-like aligning term, and the second term corresponds to the finite but large sharpness of vision-cone boundary. It is straight forward to see that similar expression can be obtained for $\hat{\mathbf{v}}_{2}(t+1)$ from Eq. 12 using the asymptotic expansion, where $\sigma_{12}$ will be replaced by $\sigma_{21}$ as follows,

$$
\hat{\mathbf{v}}_{2}(t+1) \simeq \frac{1}{2}\left(\hat{\mathbf{v}}_{2}(t)+\hat{\mathbf{v}}_{1}(t)\right)+\frac{1}{4}\left(\hat{\mathbf{v}}_{2}(t)-\hat{\mathbf{v}}_{1}(t)\right) e^{-q\left|\sigma_{21}\right|}
$$

In general $\delta_{12}$ is independent of $\delta_{21}$, and therefore $\sigma_{12} \neq$ $\sigma_{21}$. Thus, though the first term of Eqs. 1314 , i.e. the Vicsek-like term is symmetric under the exchange of the agents, the second term is not. And this is the root cause for non-reciprocity. Eq. 13 and 14 can be written in a concise way for any time-step $\Delta t$ as,

$$
\begin{aligned}
\frac{\Delta \hat{\mathbf{v}}_{i}}{\Delta t} & =\frac{\left(\hat{\mathbf{v}}_{j}-\hat{\mathbf{v}}_{i}\right)}{2 \Delta t}\left(1-\frac{1}{2} e^{-q\left|\sigma_{i j}\right|}\right) \\
& =\mathbf{F}_{i j}\left(1-\frac{1}{2} e^{-q\left|\sigma_{i j}\right|}\right)
\end{aligned}
$$

where $(i, j) \in(1,2)$ and $i \neq j$. For the above dynamics, $\mathbf{F}_{i j}=-\mathbf{F}_{j i}$ and this can be considered as the reciprocal force acting between $i^{\text {th }}$ and $j^{\text {th }}$ agents. The nonreciprocity arises from the fact that $\sigma_{i j} \neq \sigma_{j i}$. Here we like to point out that the non-reciprocity persists for arbitrarily large but finite value of the sharpenss parameter $q . q\left|\sigma_{i j}\right|$ In $q\left|\sigma_{i j}\right| \rightarrow \infty$ limit we recover the reciprocal Vicsek-like interaction. The interaction above in Eq.16 is similar to the one used to simulate a passive non-reciprocal system in 65.

When agent " 2 " is not inside the vision-cone of " 1 ", Eq. 11] becomes,

$$
\hat{\mathbf{v}}_{1}(t+1) \simeq \hat{\mathbf{v}}_{1}(t)+\left(\hat{\mathbf{v}}_{2}(t)-\hat{\mathbf{v}}_{1}(t)\right) e^{-q\left|\sigma_{12}\right|}
$$

Similar expressions can also be obtained for $\hat{\mathbf{v}}_{2}(t+1)$ from Eq.12 where instead $\sigma_{12}$, we will have $\sigma_{21}$. As $\sigma_{i j} \neq \sigma_{j i}$ in general, in this case also the system is in general nonreciprocal. In the limit of $\left(q\left|\sigma_{i j}\right|, q\left|\sigma_{j i}\right|\right) \rightarrow \infty$ the system becomes non-interacting and therefore trivially reciprocal.

\section{References}

1. D. Bi, X. Yang, M. C. Marchetti, and M. L. Manning, "Motility-driven glass and jamming transitions in biological tissues," Physical Review X, vol. 6, no. 2, p. 021011, 2016.

2. H. P. Zhang, A. Be'er, R. S. Smith, E.-L. Florin, and H. L. Swinney Euro Phys Lett, vol. 87, p. 48011, 2009.

3. N. C. Darnton, L. Tuner, S. Rojevsky, and H. C. Berg Biophys. J, vol. 98, p. 2082, 2010.

4. B. A. Camley and W.-J. Rappel, "Physical models of collective cell motility: from cell to tissue," Journal of Physics D: Applied Physics, vol. 50, no. 11, p. 113002, 2017.

5. F. Peruani, J. Starruß, V. Jakovljevic, L. SøgaardAnderson, A. Deutsch, and M. Bär Phy Rev Lett, vol. 108, p. 098102, 2012 . 
6. H. Ke, S. Ye, R. L. Carroll, and K. Showalter J. Phys. Chem. A, vol. 114, pp. 5462-5467, 2010.

7. I. Buttinoni, J. Bialké, F. Kümmel, H. Löwen, C. Bechinger, and T. Speck Phys Rev Lett, vol. 110, p. 238301, 2013.

8. A. Kudrolli, G. Lumay, D. Volfson, and L. S. Tsimring Phys Rev Lett, vol. 100, p. 058001, 2008.

9. I. S. Aranson, D. Volfson, and L. S. Tsimring Phys Rev E, vol. 75, p. 051301, 2007.

10. P. Romanczuk, M. Bär, W. Ebeling, B. Lindner, and L. Schimansky-Geier, "Active brownian particles," The European Physical Journal Special Topics, vol. 202, no. 1, pp. 1-162, 2012.

11. C. Bechinger, R. Di Leonardo, H. Löwen, C. Reichhardt, G. Volpe, and G. Volpe, "Active particles in complex and crowded environments," Reviews of Modern Physics, vol. 88, no. 4, p. 045006, 2016.

12. I. D. Couzin and J. Krause Adv Study Behav, vol. 32, p. 1, 2003.

13. M. Ballerini, N. Cabibbo, R. Candelier, A. Cavagna, E. Cisbani, I. Giardina, A. Orlandi, G. Parisi, A. Procaccini, M. Viale, and V. Zdravkovic Anim. Behav., vol. 76, p. 201, 2008.

14. C. Becco, N. Vandewalle, J. Delcourt, and P. Poncin Phys $A$, vol. 367 , p. $487,2006$.

15. A. E. Turgut, H. Çelikkanat, F. Gökçe, and E. Şahin Swarm Intell, vol. 2, pp. 97-120, 2008.

16. M. Ballerini, N. Cabibbo, R. Candelier, A. Cavagna, E. Cisbani, I. Giardina, V. Lecomte, A. Orlandi, G. Parisi, A. Procaccini, et al., "Interaction ruling animal collective behavior depends on topological rather than metric distance: Evidence from a field study," Proceedings of the national academy of sciences, vol. 105, no. 4, pp. 1232-1237, 2008.

17. J. Buhl, D. J. Sumpter, I. D. Couzin, J. J. Hale, E. Despland, E. Miller, and S. J. Simpson, "From disorder to order in marching locusts," Science, vol. 312, no. 5778, pp. 1402-1406, 2006.

18. L. Dyson, C. A. Yates, J. Buhl, and A. J. McKane, "Onset of collective motion in locusts is captured by a minimal model," Physical Review E, vol. 92, no. 5, p. 052708, 2015.

19. W. Bialek, A. Cavagna, I. Giardina, T. Mora, E. Silvestri, M. Viale, and A. M. Walczak, "Statistical mechanics for natural flocks of birds," Proceedings of the National Academy of Sciences, vol. 109, no. 13, pp. 4786-4791, 2012.

20. A. Cavagna, L. Del Castello, I. Giardina, T. Grigera, A. Jelic, S. Melillo, T. Mora, L. Parisi, E. Silvestri, M. Viale, et al., "Flocking and turning: a new model for self-organized collective motion," Journal of Statistical Physics, vol. 158, no. 3, pp. 601-627, 2015.

21. T. Vicsek, A. Czirók, E. Ben-Jacob, I. Cohen, and O. Shochet Phys. Rev. Lett, vol. 75, pp. 1226-1229, 1995.

22. H. Chaté, F. Ginelli, G. Grégoire, and F. Raynaud Phys. Rev. E, vol. 77, p. 046113, 2008.

23. G. Grégoire, H. Chaté, and Y. Tu Phys. D, vol. 181, pp. $157-170,2003$.

24. J. Toner and Y. Tu Phys Rev E, vol. 58, p. 4828, 1998.

25. H. Levine, W.-J. Rappel, and I. Cohen Phys Rev E, vol. 63, p. $017101,2000$.

26. G. Grégoire and H. Chaté Phys Rev Lett, vol. 92, p. 025702, 2004.

27. H. Chaté, F. Ginelli, G. Gregoiré, F. Peruani, and F. Raynaud Eur. Phys. J. B., vol. 64, p. 451, 2008.
28. G. Salbreux, G. Charras, and E. Paluch, "Actin cortex mechanics and cellular morphogenesis," Trends in cell biology, vol. 22 , no. 10 , pp. $536-545,2012$

29. M. Mayer, M. Depken, J. S. Bois, F. Jülicher, and S. W. Grill, "Anisotropies in cortical tension reveal the physical basis of polarizing cortical flows," Nature, vol. 467, no. 7315 , p. $617,2010$.

30. M. Behrndt, G. Salbreux, P. Campinho, R. Hauschild, F. Oswald, J. Roensch, S. W. Grill, and C.-P. Heisenberg, "Forces driving epithelial spreading in zebrafish gastrulation," Science, vol. 338, no. 6104, pp. 257-260, 2012.

31. A. Saha, M. Nishikawa, M. Behrndt, C.-P. Heisenberg, F. Jülicher, and S. W. Grill, "Determining physical properties of the cell cortex," Biophysical journal, vol. 110, no. 6, pp. 1421-1429, 2016.

32. S. R. Naganathan, S. Fürthauer, M. Nishikawa, F. Jülicher, and S. W. Grill, "Active torque generation by the actomyosin cell cortex drives left-right symmetry breaking," Elife, vol. 3, p. e04165, 2014.

33. M. Nishikawa, S. R. Naganathan, F. Jülicher, and S. W. Grill, "Controlling contractile instabilities in the actomyosin cortex," eLife, vol. 6, p. e19595, 2017.

34. T. Vicsek and A. Zafeiris Phys. Rep., vol. 517, p. 71, 2012.

35. A. Menzel Phys. Rep., vol. 554, p. 1, 2015.

36. R. D. Peacock, J. D. Averill, et al., Pedestrian and evacuation dynamics. Springer Science \& Business Media, 2011.

37. M. Mijalkov, A. McDaniel, J. Wehr, and G. Volpe, "Engineering sensorial delay to control phototaxis and emergent collective behaviors," Physical Review X, vol. 6, no. 1, p. 011008, 2016.

38. E. Forgoston and I. B. Schwartz, "Delay-induced instabilities in self-propelling swarms," Physical Review E, vol. 77, no. 3, p. 035203, 2008.

39. J. Hindes, K. Szwaykowska, and I. B. Schwartz, "Hybrid dynamics in delay-coupled swarms with mothership networks," Physical Review E, vol. 94, no. 3, p. 032306, 2016.

40. K. Szwaykowska, I. B. Schwartz, L. M.-y.-T. Romero, C. R. Heckman, D. Mox, and M. A. Hsieh, "Collective motion patterns of swarms with delay coupling: Theory and experiment," Physical Review E, vol. 93, no. 3, p. 032307, 2016.

41. J. D. Biggs, D. J. Bennet, and S. K. Dadzie, "Timedelayed autosynchronous swarm control," Physical Review $E$, vol. 85, no. 1, p. $016105,2012$.

42. U. Erdmann, W. Ebeling, and A. S. Mikhailov, "Noiseinduced transition from translational to rotational motion of swarms," Physical Review E, vol. 71, no. 5, p. 051904, 2005.

43. K. Dholakia and P. Zemánek, "Colloquium: Gripped by light: Optical binding," Reviews of modern physics, vol. 82, no. 2, p. $1767,2010$.

44. E. R. Shanblatt and D. G. Grier, "Extended and knotted optical traps in three dimensions," Optics express, vol. 19, no. 7, pp. 5833-5838, 2011.

45. B. Sabass and U. Seifert, "Efficiency of surface-driven motion: Nanoswimmers beat microswimmers," Physical review letters, vol. 105, no. 21, p. 218103, 2010.

46. R. Soto and R. Golestanian, "Self-assembly of catalytically active colloidal molecules: tailoring activity through surface chemistry," Physical review letters, vol. 112, no. 6, p. 068301, 2014.

47. J. Dzubiella, H. Löwen, and C. Likos, "Depletion forces in nonequilibrium," Physical review letters, vol. 91, no. 24, p. 248301, 2003. 
48. A. S. Khair and J. F. Brady, "On the motion of two particles translating with equal velocities through a colloidal dispersion," in Proceedings of the Royal Society of London A: Mathematical, Physical and Engineering Sciences, vol. 463, pp. 223-240, The Royal Society, 2007.

49. C. Mejía-Monasterio and G. Oshanin, "Bias-and bathmediated pairing of particles driven through a quiescent medium," Soft Matter, vol. 7, no. 3, pp. 993-1000, 2011.

50. V. N. Tsytovich, "Dust plasma crystals, drops, and clouds," Physics-Uspekhi, vol. 40, no. 1, pp. 53-94, 1997.

51. G. E. Morfill and A. V. Ivlev, "Complex plasmas: An interdisciplinary research field," Reviews of modern physics, vol. 81, no. 4, p. 1353, 2009.

52. M. Chaudhuri, A. V. Ivlev, S. A. Khrapak, H. M. Thomas, and G. E. Morfill, "Complex plasmathe plasma state of soft matter," Soft Matter, vol. 7, no. 4, pp. 1287-1298, 2011.

53. D. Helbing, I. Farkas, and T. Vicsek, "Simulating dynamical features of escape panic," Nature, vol. 407, no. 6803, pp. 487-490, 2000.

54. D. Helbing and P. Molnar, "Social force model for pedestrian dynamics," Physical review E, vol. 51, no. 5, p. 4282, 1995.

55. J. Gao, S. Havlin, X. Xu, and H. E. Stanley, "Angle restriction enhances synchronization of self-propelled objects," Physical Review E, vol. 84, no. 4, p. 046115, 2011.

56. M. Durve and A. Sayeed, "First-order phase transition in a model of self-propelled particles with variable angular range of interaction," Physical Review E, vol. 93, no. 5, p. $052115,2016$.

57. B.-M. Tian, H.-X. Yang, W. Li, W.-X. Wang, B.-H. Wang, and T. Zhou Phys. Rev. E, vol. 79, p. 052102, 2009.

58. L. Barberis and F. Peruani, "Large-scale patterns in a minimal cognitive flocking model: Incidental leaders, nematic patterns, and aggregates," Physical Review Letters, vol. 117 , no. 24 , p. $248001,2016$.

59. V. Schaller, C. A. Weber, B. Hammerich, E. Frey, and A. R. Bausch, "Frozen steady states in active systems," Proceedings of the National Academy of Sciences, vol. 108, no. 48, pp. 19183-19188, 2011.

60. Y. Sumino, K. H. Nagai, Y. Shitaka, D. Tanaka, K. Yoshikawa, H. Chaté, and K. Oiwa, "Large-scale vortex lattice emerging from collectively moving microtubules," Nature, vol. 483, no. 7390, pp. 448-452, 2012.

61. K. H. Nagai, Y. Sumino, R. Montagne, I. S. Aranson, and H. Chaté, "Collective motion of self-propelled particles with memory," Physical review letters, vol. 114, no. 16, p. $168001,2015$.

62. M. Durve, A. Saha, and A. Sayeed (Unpublished).

63. O. Chepizhko, E. G. Altmann, and F. Peruani, "Optimal noise maximizes collective motion in heterogeneous media," Physical review letters, vol. 110, no. 23, p. 238101, 2013.

64. M. E. Cates and J. Tailleur, "Motility-induced phase separation," Annu. Rev. Condens. Matter Phys., vol. 6, no. 1, pp. 219-244, 2015.

65. A. Ivlev, J. Bartnick, M. Heinen, C.-R. Du, V. Nosenko, and H. Löwen, "Statistical mechanics where newtons third law is broken," Physical Review X, vol. 5, no. 1, p. 011035 , 2015 . 